\title{
INITIATIVES D' ACTIVISME EN FORMATION INITIALE DE PROFESSEURS : PRÉPARER À L'ACTION ET À LA TRANSFORMATION
}

\author{
Elisabete Linhares \\ Escola Superior de Educação do Instituto Politécnico de Santarém \\ Unidade de Investigação e Desenvolvimento em Educação e Formação (UIDEF)- IEUL \\ elisabete.linhares@ese.ipsantarem.pt \\ Pedro Reis \\ Instituto de Educação da Universidade de Lisboa \\ Unidade de Investigação e Desenvolvimento em Educação e Formação (UIDEF)- IEUL \\ preis@ie.ulisboa.pt
}

Résumé

L'action collective raisonnée, connue sous le nom d'activisme, orientée vers la résolution de problèmes de la société, offre des possibilités d'apprentissage tout au long de la vie et permet aux étudiants de développer des connaissances du domaine de la science et de la technologie. Dans une société où les découvertes scientifiques et les progrès technologiques sont constants, les potentialités de cette approche sont également reconnues dans la formation de citoyens responsables, capables d'agir et de résoudre des problèmes, tout en essayant d'influencer d'autres citoyens à prendre des décisions sur les problèmes complexes tels que ceux que présentent les questions socialement vives. Considérant que la formation de jeunes et d'adultes plus responsables et scientifiquement informés est fondamentale, cette recherche-action visait: a) à munir les futurs enseignants de compétences dans la conception, la mise en œuvre et l'évaluation des activités orientées vers l'action sociopolitique sur les questions socialement vives; b) promouvoir chez les participants les valeurs de citoyenneté et de sensibilisation à l'environnement; c) identifier les difficultés et le potentiel de ces initiatives; d) comprendre l'impact de ce type d'expérience éducative chez les futurs enseignants. Les participants à l'étude étaient des étudiants préparant une licence en éducation élémentaire (futurs professeurs des écoles primaires et maternelles) dans le contexte de la discipline d'Éducation à l'Environnement. L'analyse des narratives produites par les futurs enseignants a permis de constater que tous 
avaient développé des compétences au niveau de la planification et de l'intervention dans la société, en recourant à différents types d'initiatives. Cette expérience de formation a ainsi permis, aux participants le développement de capacités à l'action et à la transformation sociale et environnementale. Il a également été possible de sensibiliser le public-cible des actions réalisées par les futurs enseignants à agir de façon plus responsable sur l'environnement.

Mots-Clés: Questions Socialement Vives; Action sociopolitique raisonnée; Formation initial des enseignants; Transformation social et environnemental.

\section{Abstract}

Informed collective action, also known as activism, oriented to solve society's problems, offers opportunities to life-long learning and allows students to learn about science and technology. In a society where scientific discoveries and technological advances are constant, it is still recognized the potential of this approach in the training of responsible citizens capable of acting and solving problems, and also with a view to influence other citizens in making decisions about complex issues such as those that involve social acute questions. Considering fundamental to develop more responsible and scientifically informed citizens, this action-research intended to: a) equip future teachers with the skills necessary for the design, implementation and evaluation of initiatives aimed at socio-political action on social acute questions; b) promote the participants' citizenship skills and their environmental awareness; c) identify the difficulties and potentialities of their involvement in such initiatives; and d) understand the impact of this type of educational experience in the future teachers personal and professional development. The participants involved action-research were graduation students in Elementary Education (future teachers) attending a subject on Environment. The analysis of their narratives has shown the development of planning and social intervention skills through different types of initiatives. This training experience enabled the participants for action and social and environmental transformation. It was also possible to sensitize the actions' target audiences to act more responsibly in the environment.

Keywords: Social acute issues; Informed collective action; Initial training of teachers; Social and environmental transformation. 


\section{Introduction}

Selon Fernandes, Gonçalves Pereira et Azeiteiro (2007), l'école est un lieu privilégié pour promouvoir le changement et la clarification nécessaires à-travers les valeurs environnementales, afin d'élucider les jeunes et de les rendre plus responsables. Selon ces auteurs, «l'éducation à l'environnement vise non seulement l'acquisition de contenus et de connaissances sur l'environnement, mais, avant tout, un changement de comportements et d'attitudes, conduisant à une résolution effective des problèmes environnementaux» (p. 28). Ainsi, son but ultime est de privilégier le développement de citoyens éclairés et instruits en matière d'environnement, capables de participer aux problèmes de la société dans une perspective d'amélioration de la qualité de vie (Loi ${ }^{\circ}{ }^{\circ 11 / 87}$ du 7 Avril - Droit sur les Bases de l'Environnement). La formation des enseignants joue ici un rôle fondamental, compte tenu que les étudiants d'aujourd'hui, professionnels de demain, pourront à leur tour, contribuer chaque jour, tant au niveau professionnel que personnel, à la formation de jeunes plus participatifs et responsables. Comme le soutient Hodson (2014), l'école et ses enseignants doivent offrir aux étudiants la possibilité de «travailler en groupe, assumer des responsabilités et prendre part à des activités permettant de réaliser les changements» (p. 69). L'approche de questions socialement vives est particulièrement propice à la promotion de pratiques d'intervention qui visent à la transformation sociale et environnementale, et permettant de cultiver un sens de la communauté, des relations aux autres, des devoirs et des responsabilités. C'est en montrant aux futurs enseignants comment construire une société active qu'il sera possible d'inverser la tendance, précédemment rappelée, du détachement total des citoyens par rapport aux problèmes qui affectent la société. Les initiatives d'activisme visent à élucider les étudiants et à les doter de connaissances sur certains des problèmes actuels, ce qui suscite des moments de réflexion et des attitudes critiques à l'égard des informations du quotidien et permet une prise de décision et une action responsable sur ces questions socialement vives.

\section{Encadrement théorique}

Dans une société où les conflits socio-scientifiques et socio-environnementaux sont constants, l'action sociopolitique raisonnée se présente comme une pratique éducative particulièrement intéressante et pertinente. La formation des citoyens munis de connaissances scientifiques et technologiques et de compétences de citoyenneté 
est fondamentale pour comprendre les événements qui marquent la vie quotidienne d'une société, et permet un plus grand engagement et une plus grande participation face aux problèmes sociaux (Blatt, 2014). La résolution de problèmes environnementaux peut être travaillée à partir d'initiatives d"activisme ayant pour base les questions socialement vives. L'approche des questions socialement vives est particulièrement avantageuse pour décrire la complexité et les incertitudes des problèmes environnementaux, puisqu'elle fait référence à des controverses à implications sociales (Simonneaux et Simonneaux, 2012). Pour Legardez et Simonneaux (2004), une question controversée est celle qui est "vive": a) dans la société - pour faire fréquemment l'objet d'un traitement médiatique, de telle sorte que quiconque ayant quelque connaissance sur cette question, peut susciter la discussion; b) dans les savoirs de référence - au cours de débats entre experts de différentes disciplines.

Pour contrarier une participation de plus en plus réduite des citoyens due à la complexité croissante des problèmes qui affligent la société, il est important d'investir dans la formation des enseignants où sont privilégiées les compétences nécessaires au développement d'une éducation orientée vers l'action sociopolitique (activisme) visant une société plus juste et plus durable (Hodson, 2014). Cette formation devrait permettre aux enseignants en formation initiale, de mettre en œuvre leurs valeurs et leurs convictions de manière responsable et, à leur tour, d'encourager le public-cible à développer ces mêmes capacités. Face au travail déjà accompli auprès de futurs enseignants, Bencze et Sperling (2012) affirment qu'une expérience de formation en activisme tend à influencer positivement les enseignants à adopter ces pratiques dans leur futur contexte d'intervention. La mise en œuvre de programmes orientés vers l'activisme dans tous les cours de formation d'enseignants peut s'avérer un facteur fondamental pour transposer les approches traditionnelles d'enseignement qui donnent priorité à la consommation des connaissances (Hoeg \& Bencze, 2014; Karahan \& Roehrig, 2015). Hoeg et Bencze (2014) rappellent d'autre part que les enseignants en formation initiale développent des philosophies et des approches pédagogiques. Par conséquent, les expériences de formation initiale auront une forte influence sur les pratiques qu'ils adapteront dans leur avenir professionnel. Ces pratiques auront un impact direct sur les étudiants qu'ils formeront à leur tour, et indirect dans la construction d'une société plus (ou moins) participée. II est donc important que les institutions d'enseignement supérieur et les cours de formation d'enseignants respectifs cherchent à intégrer des programmes d'action sur la base de pratiques 
sociopolitiques, contribuant ainsi à la construction d'une société plus critique, responsable et durable (Chawla, 2008). Schusler et Krasny (2015) ont constaté que les initiatives environnementales auxquelles ont participé quelques jeunes leur ont permis de développer les capacités nécessaires pour intervenir dans la société en tant que citoyens scientifiquement instruits. Ces jeunes ont été capables d'arriver à leurs propres conclusions sur les questions scientifiques, technologiques et / ou environnementales, ainsi que de négocier au niveau d'autres processus démocratiques attenants à des questions sociales, économiques, environnementales et éthiques, ce qui leur a permis de faire preuve de raisonnement critique. Lorsque les étudiants ont la possibilité de participer activement à l'amélioration de leur environnement, tout en étant eux-mêmes les agents de leur propre apprentissage, ils sont par la suite capables d'assumer des responsabilités dans leur vie et de faire face au changement (Simovska, 2008). Comme l'explique Blatt (2014), le fait que les étudiants participent à des actions activistes pendant leur formation ne signifie pas qu'ils développeront un comportement d'intervention dans leur vie quotidienne, cependant, des possibilités leur sont ainsi données de développer une «mentalité activiste» quand ils sont amenés à réfléchir sur leurs comportements et à comprendre qu'ils peuvent agir pour contribuer à la résolution de problèmes. Hodson (2014) souligne aussi l'importance d' "analyser les moyens de munir les jeunes de capacités pour faciliter la solution des problèmes de la société au lieu de contribuer à leur existence.» (p.64). L'auteur fait également référence à l'importance des actions orientées dans le contexte d'enseignement des Sciences, dans la mesure où les étudiants y obtiennent une meilleure compréhension des questions socialement vives auxquelles ils sont confrontés, notamment leur dimension sociopolitique et éthique. Une autre contribution se centre sur l'engagement que peuvent prendre les étudiants face au développement d'actions sociopolitiques, aussi bien individuellement que collectivement. Dans un programme fondé sur des initiatives d'activisme, plus encore que les valeurs et les idées subjacentes au problème en étude, il est important de vivre des expériences d'apprentissage qui engagent les étudiants dans des situations d'action sociopolitiques. Hodson (2014) attire l'attention sur le fait qu'il est beaucoup plus facile à quiconque de parler de ses préoccupations vis à vis d'une question plutôt que de faire quoi que ce soit pour la résoudre d'une manière cohérente et efficace. En effet, en plus de devoir changer notre comportement, il nous faut prendre des mesures pour changer le comportement des autres. C'est uniquement ainsi qu'une pression pourra être exercée sur les prises de décision politique, et que la parole sera prise 
dans le but d'établir la justice sociale ou d'arrêter la dégradation de l'environnement dans le contexte des questions socialement vives. La transformation sociale et environnementale que peut déclencher chacun de nous est fondamentale dans une société qui se veut démocratique.

Bien que plusieurs auteurs reconnaissent les avantages de l'action sociopolitique dans la formation des étudiants pour la résolution démocratique des problèmes sociaux (Bencze \& Sperling, 2012; Conde, 2014; Hodson, 2014; Karahan \& Roehrig, 2015; Linhares \& Reis, 2014; Reis, 2014e, 2014b; Roth, 2009), il existe des obstacles à son adoption en classe. Reis (2014) présente quelques prémisses fondamentales pour l'intégration des pratiques orientées vers l'activisme, notamment: a) croire au potentiel éducatif de cette approche pour les étudiants; b) avoir connaissance des interactions entre la science, la technologie, la société et l'environnement; c) avoir des connaissances pédagogiques sur la mise en place d'initiatives d'activisme; d) avoir la volonté et être capables de changer la culture de l'école et de la société. Une autre condition fondamentale pour la réussite des initiatives d'activisme environnemental à l'école tient à l'existence d'atouts permettant aux étudiants et aux enseignants de s'engager dans des situations d'activisme ainsi qu'à la disponibilité d'enseignants prêts à accompagner les étudiants dans ces projets tout au long de l'année (Kerchkhoff \& Reis, 2014).

Comme dans l'étude de Bader et Laberge (2014), notre intention était, d'une part, de donner un sens aux apprentissages des futurs enseignants, compte tenu des réelles préoccupations attenantes aux questions qui affectent leur vie quotidienne et, d'autre part, de les préparer à l'action conduisant à la résolution de ces problèmes et à la protection de l'environnement. Ainsi, chaque groupe de futurs enseignants a eu la possibilité de choisir un problème à travailler. Selon Hodson (2014), apprendre à développer une action sociopolitique passe par trois étapes:

- Modélisation - l'enseignant démontre et explique le comportement souhaité (dans ce cas, l'activisme social) et fournit des exemples illustratifs.

- Pratique guidée - les étudiants effectuent des tâches spécifiques au sein d'une action globale avec l'aide de l'enseignant.

- Application - les étudiants travaillent / agissent indépendamment de l'enseignant. (p.87)

Hodson (2014) considère que les étudiants apprennent à planifier, à mettre en œuvre et à évaluer des actions sociopolitiques en observant la façon dont les enseignants s'engagent dans l'action, en pratiquant des compétences, en recourant à 
des simulations de situations et en comptant sur l'appui et le feed-back du professeur qui favorise réflexion sur l'activité en cours au sein de chaque groupe. Dans une étude menée par Lester, Ma, Lee et Lambert (2006), auprès d'étudiants de l'enseignement primaire âgés de 10 à 11 ans, ceux qui avaient une connaissance scientifique plus approfondie sur le sujet d'étude (effet de serre et réchauffement global), s'exprimaient plus fréquemment en termes d'activisme social. Cependant, les auteurs n'ont pas analysé les pratiques des étudiants afin de vérifier si cette prise de conscience sociale de l'activisme représentait une manifestation individuelle responsable ou se traduisait au niveau de l'action collective. Dans la présente étude, nous avons cherché à aller plus loin, essayant de donner aux futurs enseignants en formation l'occasion d'influencer les autres (public-cible de leur choix, famille, voisins, amis, enfants ou entreprises/ institutions).

\section{Méthodologie}

La présente étude c'est centrée sur une recherche-action. La recherche-action est considérée comme un dispositif où les activités éducatives et les processus de recherche se produisent simultanément. La recherche accompagne l'action vers sa compréhension. Les connaissances qui en découlent sont réinvesties dans l'action elle-même puisqu'elle vise à la transformation. II s'agit donc d'un processus de recherche sur l'action, par l'action et pour l'action, avec la participation active des auteurs de cette action (Caetano, 2004). Pazos (2002) dégage comme principales caractéristiques de cette méthodologie de recherche: la connaissance, l'action, l'amélioration et la collaboration. Pour l'auteure, la recherche-action vise à agir sur des actions et des situations inhérentes à l'environnement naturel de la classe. L'association des termes action et recherche met en évidence les principales caractéristiques de cette méthode: la mise en œuvre et l'évaluation des idées afin de développer la connaissance et améliorer le programme d'études, l'enseignement et l'apprentissage (Kemmis \& McTaggart, 1982). Ainsi, considérant fondamentale l'adoption de pratiques éducatives qui permettent aux futurs enseignants en formation une plus grande participation dans leur processus d'enseignement-apprentissage et le développement d'une attitude plus critique et d'intervention au quotidien, nous avons essayé de mieux comprendre la façon dont les activités centrées sur l'action sociopolitique pouvaient permettre d'atteindre ces objectifs. 
Cette recherche-action réalisée dans le contexte d'une discipline d'Environnement auprès de deux classes de licence en enseignement élémentaire visait à: a) doter les futurs enseignants de compétences au niveau de la conception de la mise en œuvre et de l'évaluation d'activités orientées vers l'action sociopolitique sur les questions socialement vives; b) promouvoir chez les participants des compétences de citoyenneté et une prise de conscience de l'environnement; c) identifier les difficultés et le potentiel de ce genre d' initiative; d) comprendre l'impact de ce type d'expérience éducative chez les futurs enseignants.

La collecte des données a été réalisée à partir de récits produits (narratives) en fin du semestre, par les étudiants qui avaient animé les activités d'activisme prévues. Une narrative permet de comprendre un contexte particulier, dans le cas concret, l'expérience éducative vécue par ce groupe de futurs enseignants. Comme l'indiquent Galvão (2005), Reis (2008) et Kings et Climent (2012), la narrative, en tant que processus d'interaction entre les chercheurs et les auteurs, permet, d'une part, de comprendre les causes, les intentions et les objectifs de certaines actions, et d'autre part, d'accéder à une meilleure connaissance et compréhension d'une réalité sociale. Reis $(2004,2008)$ ajoute également que la discussion (entre chercheurs et auteurs) des récits ainsi construits ouvre l'accès aux idées des auteurs, de sorte que leur analyse permet à l'enseignant-chercheur de l'étude de considérer les initiatives de l'action sociopolitique de manière réflexive. Cette analyse réflexive peut mener l'enseignant au changement dans la conception, l'orientation et l'organisation de ces actions pour promouvoir des activités plus significatives chez les étudiants. Le discours des narrateurs, obtenu sous forme de texte, a été considéré en fonction des objectifs de la recherche, ce qui nous a permis d'accéder aux perspectives des futurs enseignants sur l'expérience éducative vécue, centrée sur les actions sociopolitiques qu'ils avaient eux-mêmes mises en place. Les données présentées dans les récits ont été soumises à une analyse de contenu catégorielle, afin de rendre plus compréhensible le matériel collecté et de permettre son interprétation. Le processus de codage devait tenir à l'émergence de régularités et de modèles rencontrés dans les récits. Les modèles ainsi retrouvés ont permis de créer des catégories en fonction des objectifs de l'enquêteur, ce qui représente un moyen de classer les données descriptives recueillies (Bardin, 2009). Pour compléter l'analyse qualitative, nous avons procédé, en outre, à une analyse quantitative pour détecter les tendances. Les interprétations de l'enseignant-chercheur, résultant de l'analyse de contenu sont accompagnées de quelques extraits narratifs. Ces extraits ne constituent pas une 
narrative mais résument et appuient les déclarations produites. Comme nous l'avons mentionné précédemment, les thèmes des travaux sont centrés sur les questions socialement vives, ce qui implique plusieurs dimensions, telles que les dimensions scientifique, technologique, sociale et environnementale. Du fait qu'ils soient issus de différents secteurs, les problèmes en étude sont complexes et requièrent l'analyse et la compréhension des différents participants. Les futurs enseignants ont formé des groupes de travail et ont déterminé la question environnementale à aborder ainsi que la forme d'action sociopolitique à réaliser. La tâche a débuté par une phase de familiarisation à partir d'exemples de différentes initiatives d'action sociopolitique, suivie de la discussion et de la réflexion par l'ensemble de la classe, sur la pertinence de ce type d'intervention dans la société et de la recherche du matériel sur le thème choisi. Comme le déclare Hodson (2014), avant d'engager les étudiants dans l'action sociopolitique proprement dite, nous considérons qu'il est essentiel de les doter de connaissances scientifiques sur les QSV à travailler. Ainsi, une première étape a été constituée par une recherche des problématiques en analyse afin de mieux comprendre la complexité du thème et de reconnaître les positions concernées pour pouvoir ensuite prendre une décision responsable face à une question socialement vive. Un autre moment a permis à tous les groupes de concevoir une affiche en ligne (utilisation du logiciel Glogster) sur la problématique abordée dans chaque groupe. Finalement, tous les groupes ont planifié et mis en place une forme d'activisme de leur choix. Ils ont également déterminé le public-cible auquel elle se destinait. Ce travail s'est déroulé tout au long du second semestre scolaire 2013-2014.

\section{Présentation des résultats et discussion}

Les questions socialement vives étudiées par les différents groupes de travail étaient diversifiées et intégraient des dimensions économiques, politiques, environnementales et sociales dans chacun des problèmes. Les thématiques abordées en plus grand nombre se rapportaient au changement de climat $(24,1 \%)$, à la déforestation $(20,7 \%)$ et à la consommation excessive des ressources naturelles $(10,3 \%)$. Les problématiques environnementales sélectionnées par les étudiants se centraient sur des thèmes actuels et de leur quotidien qui les préoccupaient et sur lesquels ils souhaitaient avoir plus de connaissances pour pouvoir divulguer les valeurs environnementales auprès du public-cible des initiatives prévues. 
Les destinataires des actions mises en place par les futurs enseignants ont été répartis en groupes dans lesquels prédominaient les enfants de l'enseignement primaire $(20,7 \%)$, de la maternelle $(13,8 \%)$, des centres de loisirs - Centres d'Activités de Temps Libres (CATL) - ainsi que les citoyens de la communauté en général (10,3\% dans chaque groupe). Le choix des participants à cette étude avait été surtout fixé en fonction du profil professionnel des futurs enseignants, ceux-ci ayant opté en faveur de destinataires de différents niveaux de scolarité, à savoir enseignement préscolaire (école maternelle) et 1er cycle éducation de base (école primaire). D'autre part, quelques références devaient justifier certains choix guidés par la volonté de promouvoir des valeurs environnementales parmi les plus jeunes:

Le thème des changements de climat nous concernant tous, rien de mieux que d'aborder ce sujet avec les enfants car s'ils apprennent précocement à prendre conscience de la question, il leur sera plus facile de prendre des mesures et parfois même d'enseigner à leurs parents l'importance d'une planète plus "saine » (Narrative, $8 D)$

Il faudrait commencer à mettre en œuvre ce type d'action chez les jeunes enfants (Narrative, $6 P L$ )

Pour les enseignants en formation, les principales finalités de l'action collective réalisée consistaient à: a) divulguer l'information (55,2\%); b) sensibiliser les destinataires de ces actions $(44,8 \%)$; c) améliorer l'environnement (37,9\%); et d) prendre des mesures d'intervention $(27,6 \%)$. D'autres finalités ont été mentionnées moins fréquemment, notamment : changer de perspective $(3,5 \%)$ et développer une opinion critique (10,3\%) (Tableau 1).

Compte tenu de la nature des thèmes exploités dans les actions collectives réalisées, un grand nombre d'affirmations considère la sensibilisation du public-cible comme principale finalité de l'initiative, afin de promouvoir la conscience environnementale, la capacité de réflexion et, finalement, l'adoption de pratiques visant à améliorer le problème de l'environnement abordé. Parallèlement, quelques considérations renvoient à la divulgation d'informations sur le thème, qui permettraient aux participants à l'action de savoir comment minimiser un problème environnemental particulier et ainsi contribuer à une amélioration de l'environnement :

Sensibiliser les participants, faire en sorte qu'ils développent leur conscience environnementale et finalement, les amener à prendre des mesures pour minimiser ce problème. (Narrative, 9D) 
Faire découvrir le thème aux enfants. Aussi bien que stimuler l'opinion critique sur le thème. Ce type d'initiative, comme celle réalisée dans mon groupe, cherche de manière ludique à fournir aux enfants des informations sur certains thèmes parfois polémiques, à leur permettre de formuler eux-mêmes leur opinion et à pouvoir discuter du sujet. (Narrative, 3PL)

Tableau 1 - Finalités attribuées par les futurs enseignants à l'action sociopolitique réalisée.

\begin{tabular}{ccc}
\hline Catégorie & $\begin{array}{c}\mathbf{N} \\
\text { (Total de } \\
\text { répondants) }\end{array}$ & Pourcentage (\%) \\
\hline $\begin{array}{c}\text { Divulguer } \\
\text { l'information }\end{array}$ & 16 & 55,2 \\
\hline $\begin{array}{c}\text { Changer de } \\
\text { perspective }\end{array}$ & 1 & 3,5 \\
\hline $\begin{array}{c}\text { Sensibiliser le public- } \\
\text { cible }\end{array}$ & 13 & 44,8 \\
\hline $\begin{array}{c}\text { Prendre des } \\
\text { mesures/ Intervenir }\end{array}$ & 4 & 27,6 \\
\hline $\begin{array}{c}\text { Développer l'opinion } \\
\text { critique }\end{array}$ & 3 & 10,3 \\
\hline $\begin{array}{c}\text { Améliorer } \\
\text { l'environnement }\end{array}$ & 11 & 37,9 \\
\hline Total de Participants & $\mathbf{2 9}$ & \\
\hline
\end{tabular}

Certaines des idées exprimées par les étudiants semblent révéler une expérience dans leur formation encore très centrée sur la transmission de contenus. Chacun sait que la plupart des expériences d'enseignement vécues par les étudiants finissent par être reproduites dans la pratique de nombreux professionnels. II est donc essentiel d'intégrer des pratiques d'enseignement-apprentissage plus actives permettant aux étudiants de produire des connaissances, ainsi que le permettent les activités d'action sociopolitique. Le discours des futurs enseignants est encore très centré sur l'action de l'enseignant: «(...) montrer aux enfants les différents problèmes ... intérioriser cette matière" - Narrative, 2D; "(...) transmettre à ces enfants quelques connaissances sur ce thème" - Narrative, 13PL.

Confrontés au besoin de concevoir et mettre en œuvre une action sociopolitique, les futurs enseignants ont dû faire face à de nombreux défis. En plus du choix du thème et des destinataires de l'action, les enseignants en formation ont été encouragés à penser et à choisir une forme d'intervention. Face à cette situation ils ont 
dégagé des idées et formulé des possibilités d'action. Tous les groupes ont envisagé de façon différente la manière d'agir vis à vis de la société, et, dans certains cas, le type d'action initialement pensée n'a pas correspondu à ce que le groupe a effectivement accompli. De l'ensemble des initiatives d'abord envisagées, nous rappelons ici celles qui n'ont pas été réalisées: a) une annonce à la radio, dont le but était de présenter la problématique suivie de quelques solutions permettant aux auditeurs de réfléchir sur le thème, d'adopter des comportements plus durables, et de commencer à faire partie de la solution et non plus du problème; b) une visite de terrain en forêt pour sensibiliser les participants à l'action sur l'importance de cet écosystème et de détecter certains problèmes qui affectent la forêt afin pouvoir collecter les déchets et exploiter des moyens pour aider à sa conservation; et c) une action de nettoyage de plage. Les raisons évoquées par les groupes se sont prisent aux faits suivants, dans le cas de l'annonce radio, le groupe avait pensé à ce moyen de diffusion et action par qu'il considérait que le message parviendrait à un public plus vaste. Cependant, aucune réponse n'étant parvenue de la part des responsables de la station de radio, le groupe a dû changer sa forme d'action. La sortie en forêt s'est avérée irréalisable en raison des procédures attenantes à la dispense de cours de quelques classes et au moyen de transport nécessaire au déplacement des participants. Mêmes difficultés rencontrées dans la promotion du nettoyage de la plage. Le groupe de travail chargé de cette question devrait avoir une certaine disponibilité, assurer les moyens de transport jusqu'à la plage, ainsi que les coûts associés. Les extraits suivants mettent ces situations en évidence :

Avant d'avoir cette idée finale, nous avons pensé réaliser notre action d'activisme dans un bois ou une forêt mais cela n'a pas été possible pour des raisons de procédure et la distance de l'école (...). (Narrative, 4D)

Comme forme de sensibilisation nous aurions aimé réaliser un nettoyage de plage, mais comme cela représentait certains coûts pour une grande partie du groupe, cela n'a pas été possible. (Narrative, 12D)

En alternative nous avions la divulgation à la radio (...) un moyen pouvant réellement atteindre plus de monde, mais nous n'avons pas non plus obtenu de réponse. (Narrative, 4PL)

Les étudiants étant peu habitués aux initiatives d'activisme et peu conscients de toutes les exigences nécessaires à leur réalisation, il leur a été difficile de planifier leur forme d'intervention. La planification de l'action sociopolitique a conduit les futurs 
enseignants à développer des compétences de base pour programmer une action et agir sur la société afin de promouvoir des transformations sociales et environnementales. II est crucial d'approprier l'action aux possibilités du groupe d'intervention, en tenant compte des ressources nécessaires, des destinataires, du local, du temps disponible, parmi les facteurs fondamentaux pour la bonne gestion de l'activité. Malgré cette situation, de nombreux groupes de travail sont parvenus à concrétiser l'action sociopolitique initialement pensée, à savoir: a) présentation d'une bande dessinée dans une classe d'école primaire ; b) préparation d'une histoire pour les enfants de la maternelle; c) développement et exploitation d'une vidéo de sensibilisation; d) utilisation de facebook; e) élaboration des paroles d'une chanson; f) réalisation d'une séance de sensibilisation. Les autres initiatives sociopolitiques telles que l'initiative de bénévolat et la publication d'un article dans le journal local sont nées de l'impossibilité à réaliser les idées initiales. En ce qui concerne cette dernière intervention, le groupe semble ne pas avoir été pleinement satisfait, considérant que la publication dans le journal local ne parvient pas à un public assez vaste, cependant ce même groupe semble avoir développé une sensibilisation à l'environnement quand il estime que l'important est d'intervenir et d'essayer de faire quelque chose pour l'environnement (le groupe a envisagé une publication dans un journal national, mais le coût s'est avéré trop élevé):

En troisième alternative,- dans un Journal, nous n'avons obtenu qu'un journal local où l'information ne parvient pas à tout le monde mais, dans l'ensemble, nous pensons au moins avoir alerté quelques personnes, nous devons commencer quelque part, si nous ne faisons rien, nous n'obtiendrons pas de résultats positifs quant aux améliorations de notre planète. (Narrative, 4PL)

Selon certains enseignants en formation, les expériences proposées ont été bien accueillies par les destinataires, ceux-ci ayant manifesté intérêt, curiosité et motivation («Les enfants ont très bien répondu à ce qui leur était demandé. Ils se sont montrés curieux et participatifs. »- Narrative, 6PL) notamment, en posant des questions lors de la conférence à laquelle ils ont assisté ("Les classes ont abordé les pompiers à plusieurs reprises, posant des questions pertinentes et même sur des curiosités qu'ils voulaient satisfaire » - Narrative, 2PL). Selon certains participants, l'intervention semble également avoir marqué le public-cible («La musique a été l'une des choses dont les enfants se souviennent encore aujourd'hui» - Narrative, 1PL). 
En général, selon l'opinion des futurs enseignants, l'un des grands avantages associés aux projets d'action sociopolitique correspond à la possibilité de développer diverses capacités chez les participants. Ces capacités ont été organisées selon les dimensions suivantes: a) action de sensibilisation (65,50\%); b) processus d'enseignement-apprentissage (20,70\%); c) intervention sociale (13,80\%); e) promotion de la réflexion (3,50\%); et f) promotion des valeurs (3,50\%) (tableau 2 ).

Tableau 2 - Potentialités de l'action sociopolitique selon la perspective des futurs enseignants

\begin{tabular}{ccc}
\hline Catégorie & $\begin{array}{c}\mathbf{N} \\
\text { (Total de } \\
\text { répondants) }\end{array}$ & Pourcentage (\%) \\
\hline $\begin{array}{c}\text { Action de } \\
\text { sensibilisation }\end{array}$ & 19 & 65,5 \\
\hline $\begin{array}{c}\text { Processus } \\
\text { d'enseignement- } \\
\text { apprentissage }\end{array}$ & 6 & 20,7 \\
\hline $\begin{array}{c}\text { Intervention dans la } \\
\text { société }\end{array}$ & 4 & 13,8 \\
\hline $\begin{array}{c}\text { Promotion de la } \\
\text { réflexion }\end{array}$ & 1 & 3,5 \\
\hline $\begin{array}{c}\text { Promotion des } \\
\text { valeurs }\end{array}$ & 1 & 3,5 \\
\hline Total de Participants & $\mathbf{2 9}$ & \\
\hline
\end{tabular}

Pour $65,5 \%$ des participants, l'une des valeurs ajoutées à ces activités consiste en la sensibilisation des populations au problème en question. Comme il s'agit de projets qui requièrent un engagement et une participation active de leur part, ces populations découvrent les mesures de minimisation et les formes de résolution des problèmes. Les connaissances contribuent à accroître la préoccupation et la conscience de ce que chacun peut et doit faire pour préserver l'environnement. Le public-cible de ces actions a conscience de la façon dont on porte atteinte à l'environnement et, par la réflexion (3,5\%), les destinataires des actions comprennent le rôle qu'ils pourront avoir sur les écosystèmes afin de contribuer à minimiser les problèmes environnementaux. En conséquence, ces actions contribuent à la formation de citoyens plus participatifs $(13,8 \%)$ et plus responsables face aux applications de la science et de la technologie et leurs impacts sur la société et l'environnement. 
Ce type d'actions est très important (...) car il éveille l'attention de la population sur divers problèmes, en favorisant quelque peu la prise conscience et en faisant réfléchir un peu plus la population sur son rôle et sur les actions qu'elle peut réaliser pour protéger la planète. (Narrative, 7D)

(...) Le Cours a vraiment éveillé notre attention sur l'importance de conserver notre environnement et que nous en sommes responsables. (Narrative, 3D)

Cette initiative a été très fructueuse et intéressante, quand nous pouvons aider et encourager les gens à améliorer leur mode de vie et leur donner des raisons pour qu'ils commencent à agir, recyclage, marche à pied, utilisation d'ampoules économiques, utilisation des transports publics entre autres. (Narrative, 4PL)

Pour de nombreux enseignants en formation, cette expérience de formation a également permis de connaître une autre façon d'aborder et de travailler des concepts et des contenus scientifiques avec les enfants (processus d'enseignementapprentissage, 20,7\%). En recourant à l'action sociopolitique, il est possible d'inculquer des valeurs de préservation de l'environnement et favoriser la connaissance sur divers thèmes d'une manière plus motivante et plus efficace. La promotion de valeurs environnementales est considérée en articulation avec leur future pratique professionnelle, car ils considèrent important d'éduquer les enfants afin de provoquer en eux des changements de comportement ainsi qu'un sentiment de valorisation des actions de chacun. Parallèlement, ce type d'intervention a permis aux futurs enseignants la mise en pratique de ce qu'ils avaient appris en théorie, comme ils le déclarent :

Cette initiative est importante parce que nous devons mettre en place les connaissances chez les enfants car ils sont notre avenir, nous devons donc leur inculquer des valeurs afin de préserver l'environnement qui nous manque pour vivre. Les enfants sont notre avenir et celui de la planète aussi. (Narrative, 12PL)

Cette initiative m'a aidé parce qu'il y a une différence entre, aborder les questions au niveau de la théorie et postérieurement dans la pratique. Dans la pratique, nous prenons contact direct avec les situations et nous sommes sur le terrain et ainsi nous apprenons à réaliser ce que nous savions en théorie (Narrative, 4D)

Relativement aux apprentissages, les futurs professeurs ont considéré que ce type d'expérience éducative est promoteur de diverses compétences (en eux-mêmes): a) connaissance substantive (24,1\%); b) sensibilisation à l'environnement $(20,7 \%)$; c) connaissances didactiques (17,2\%); d) connaissance des formes d'activisme (10,3\%); 
e) travail en groupe et communication (3,5\%); et f) raisonnement (3.5). Tout le travail réalisé a favorisé la compréhension des différentes dimensions concernées dans les questions socialement vives étudiées, y compris l'identification des avantages et des inconvénients, des causes du problème, des conséquences et des mesures de minimisation. Les enseignants en formation initiale sont parvenus à construire une connaissance approfondie sur des thèmes qu'ils ignoraient ou qui semblaient ne pas les préoccuper, pour devenir des citoyens plus conscients capables d'intervenir dans la société, en éprouvant le besoin de changer de comportements.

En faisant ce travail, j'ai appris beaucoup de choses dont j'ignorais l'existence. maintenant. je suis sensibilisée et j'essaie de ne pas faire certaines erreurs comme avant. (Narrative, 8D)

(...) Nous avons fait plusieurs apprentissages sur le thème à exploiter et aussi sur l'importance des actions d'activisme en matière d'éducation. Nous avons appris par exemple, plus en détail, en quoi consistent la biodiversité, les dangers d'extinction des espèces et leurs conséquences, nous avons également compris les nombreux intérêts économiques et politiques d'où adviennent souvent des intérêts prioritaires en vertu de la biodiversité de notre planète. (Narrative,11PL)

Du point de vue didactique, certains professeurs en formation ont pondéré la réalisation d'initiatives d'activisme dans leur avenir professionnel, reconnaissant la valeur des pratiques pédagogiques de l'action, visant l'amélioration de l'environnement. Les différents types d'interventions qui peuvent être mis en œuvre constituent un facteur attractif dans ce genre d'activités et peuvent promouvoir la participation des enfants en faisant appel à des formes créatives de l'action dans la société.

J'ai beaucoup appris avec la réalisation de tous les travaux de la discipline, et j'ai particulièrement aimé le produit final des travaux, à l'avenir, j'espère pouvoir réaliser d'autres travaux abordant des questions telles que la protection de l'environnement. (Narrative, 14PL)

Savoir comment interagir avec les enfants et la possibilité de présenter une matière de manière plus créative. (Narrative, 15D)

Ces travaux ont également favorisé le travail de groupe et les capacités de communication entre participants «cela nous aide nous-mêmes dans la mesure où nous avons développé plusieurs capacités pour travailler en groupe et pour 
communiquer 》 (Narrative, 12D), et ont développé le raisonnement des futurs enseignants " l'action d'activisme nous a aidés à mieux le connaître [le thème] et nous avons aussi des opinions plus claires » (Narrative, 3PL).

Un grand nombre de futurs enseignants participant à cette étude a qualifié cette expérience éducative comme différente parce qu'elle a incité les groupes de travail à «agir», en valorisant le fait de ne pas se limiter à la théorie. L'action sociopolitique réalisée a incorporé les connaissances théoriques et est allée plus au-delà, ce qui a conduit les futurs enseignants à penser à une forme d'intervention dans la société (transformation sociale et environnementale) et à faire passer un message, à amorcer un changement de comportements, leur permettant à eux-mêmes un changement de posture face aux problèmes environnementaux. Ils ont également mis en évidence l'impact de ces initiatives dans leur formation.

En comparaison à d'autres activités menées au cours des années précédentes, je pense que cela a été beaucoup plus bénéfique parce que c'est quelque chose qui nous a fait agir et n'est pas resté seulement à la partie théorique. (Narrative, 9D)

En ce qui concerne l'activité faite par rapport à tous les autres cours de ma formation, celle-ci a eu pour moi beaucoup plus d'impact parce qu'elle a été réellement mise en place et n'est pas restée juste et que «sur le papier». (Narrative, 13D)

II s'agit donc d'activités qui vont au-delà de la salle de classe, visant un publiccible différent afin de mobiliser la société au nom d'une relation plus saine avec l'environnement.

Je n'avais jamais fait un travail d'action d'activisme du domaine des sciences et je peux voir que c'est un travail différent parce que nous ne devons pas réaliser simplement un travail écrit à présenter au professeur, mais nous pouvons aussi atteindre d'autres personnes avec cette problématique et souvent, créer des projets avec d'autres institutions. (Narrative, 5D)

Cette initiative a été unique en Sciences parce qu'elle a permis de faire participer d'autres publics au-delà de la communauté scolaire. (Narrative, 9PL)

Lors de la mise en œuvre de l'action sociopolitique, les enseignants en formation ont ressenti certaines difficultés liées à: a) la gestion de l'activité; b) la gestion du travail de groupe; c) la détermination du type d'action à entreprendre; et e) la gestion du temps (tableau 3). À noter cependant, qu'un pourcentage considérable d'étudiants $(27,6 \%)$ n'a senti aucune difficulté face à l'activité. 
Tableau 3 - Difficultés rencontrées par les futurs enseignants lors de la mise en œuvre de l'action sociopolitique.

\begin{tabular}{|c|c|c|c|}
\hline Catégorie & Sous-catégorie & $\begin{array}{c}\mathrm{N} \\
\text { (Total de } \\
\text { répondants) }\end{array}$ & Pourcentage (\%) \\
\hline \multirow{4}{*}{ Gestion de l'activité } & \multirow{4}{*}{$\begin{array}{c}\text { Tâches concernées } \\
\text { Coûts } \\
\text { Publics-cibles } \\
\text { Recherche de } \\
\text { sources }\end{array}$} & 4 & 13,8 \\
\hline & & 4 & 13,8 \\
\hline & & 7 & 24,1 \\
\hline & & 1 & 3,5 \\
\hline $\begin{array}{c}\text { Gestion du travail en } \\
\text { groupe }\end{array}$ & & 5 & 17,2 \\
\hline $\begin{array}{l}\text { Determination du } \\
\text { type d'action }\end{array}$ & & 4 & 13,8 \\
\hline Gestion du temps & & 2 & 6,9 \\
\hline Sans difficulté & & 8 & 27,6 \\
\hline Total de Participants & & 29 & \\
\hline
\end{tabular}

Les plus grandes difficultés rencontrées par les enseignants en formation se rapportent à la gestion de l'activité. En effet, certains ont déclaré avoir eu des difficultés pour cibler les destinataires de leur action, adapter leur intervention au public-cible et obtenir un nombre raisonnable de participants $(24,1 \%)$ :

"Maintenant, nous allons montrer à qui?", Comme il s'agit d'une forme d'activisme cela devrait atteindre "quelqu'un". (Narrative, 10PL)

Une autre difficulté a été de simplifier le thème pour que les termes utilisés soient facilement compris par les enfants. (Narrative, 3D)

Les principales difficultés que nous avons ressenties c'est, sans aucun doute le fait de réunir un nombre important de participants parce que la grande majorité des étudiants avait beaucoup de travail. (Narrative, 9D)

Il y a également eu des problèmes d'organisation et de manque de compétences de travail de groupe au sein même des groupes (17,2\%). Parfois, il y avait des difficultés à travailler en groupes parce qu'il semblait impossible de concilier la disponibilité de tous les membres du groupe. Le manque de compétences interpersonnelles et de gestion de petits groupes faisait obstacle au bon fonctionnement du groupe. Pour que tous travaillent en fonction d'un objectif commun - le succès du groupe -, il est essentiel que tous soient conscients de devoir fournir un 
effort pour que le groupe réussisse. La contribution de tous est importante et doit être valorisée, et la communication basée sur le respect et l'écoute de l'autre doit prévaloir :

La difficulté, c'était de faire bouger une partie de groupe en faveur du groupe lui-même, c'est à dire, faire en sorte que certains membres se rendent disponibles pour sa réalisation (de l'action). (Narrative, 13D)

Pour moi, en particulier le travail de groupe [la difficulté], pour la première fois j'ai senti qu'il n'y avait pas d'union mais des malentendus, à chaque opinion donnée il y avait un membre du groupe qui n'était pas d'accord et ainsi, il a été difficile de travailler "en groupe". (Narrative, 7PL)

Certains groupes ont connu des difficultés dans la réalisation de certaines tâches $(13,8 \%)$ liées à l'action sociopolitique à mettre en œuvre, notamment l'obtention du feedback auprès des institutions concernées, relativement aux demandes d'autorisation pour la réalisation de l'initiative avec des enfants, ou encore l'absence de réponse à un groupe qui avait essayé de publier une information dans un journal national. II faut également souligner que le choix de l'action sociopolitique par les groupes de travail n'a pas été facile $(13,8 \%)$, très probablement en raison de leur manque d'expérience au niveau de ce type d'activité. Cette situation a été cependant, surmontée.

Les plus grandes difficultés que le groupe a ressenties étaient dans la planification de l'action, parce que nous n'avions jamais fait ce genre de chose et nous sentions une grande responsabilité parce que nous voulions que tout se passe pour le mieux et que tout le monde qui ait participé puisse aimer. (Narrative, 10D)

Pour la partie de l'activisme notre groupe avait initialement quelques difficultés, mais "tout est devenu plus clair." (Narrative, 12PL)

Tous les enseignants en formation ont l'intention d'utiliser les initiatives d'action sociopolitiques dans leur future pratique professionnelle (tableau 4). Un pourcentage élevé $(48,3 \%)$ estime qu'il est fondamental de travailler les questions environnementales dès les niveaux de l'enseignement élémentaire, en ayant recours à ces pratiques éducatives car elles sont plus motivantes. Elles se présentent de manière plus attrayante pour apprendre ce qui contribue à former des citoyens plus participatifs.

Je veux suivre la filière d'enseignement à l'école maternelle, nul doute que j'ai en perspective la mise en œuvre ce type de sensibilisation dans ma future pratique 
professionnelle pour prévenir les enfants des divers problèmes qui peuvent survenir sur la planète et le rôle que chaque citoyen peut jouer dans sa prévention. (Narrative, $7 D$ )

Les objectifs ne passent pas juste par enseigner ou tester quelque chose, mais aussi par la sensibilisation (des enfants) pour qu'ils deviennent des adultes socialement actifs et intégrés dans la société. L'utilisation de ce type d' initiatives est toujours importante dans la pratique professionnelle. (Narrative, $3 P L$ )

Tableau 4 - Opinion des futurs enseignants quant à l'utilisation possible d'initiatives d'action sociopolitique dans leur pratique professionnelle future.

\begin{tabular}{|c|c|c|c|}
\hline Catégorie & Sous-catégorie & $\begin{array}{c}\mathrm{N} \\
\text { (Total de } \\
\text { répondants) }\end{array}$ & Pourcentage (\%) \\
\hline \multirow{2}{*}{$\begin{array}{l}\text { Volonté de } \\
\text { promouvoir des } \\
\text { actions socio- } \\
\text { politiques } \\
\end{array}$} & $\begin{array}{l}\text { Importance pour la } \\
\text { culture des citoyens }\end{array}$ & 13 & 44,8 \\
\hline & $\begin{array}{l}\text { Importance pour la } \\
\text { formation des enfants }\end{array}$ & 14 & 48,3 \\
\hline Total de Participants & & 29 & \\
\hline
\end{tabular}

Pour un grand nombre de participants, ce type d'intervention dans la société est important pour la culture des citoyens (44,8\%). Ces initiatives présentent des valeurs de citoyenneté et de participation fondamentale dans la promotion de bonnes pratiques environnementales essentielles dans les sociétés modernes.

(...) Je conclus que c'est un atout pour ma future pratique professionnelle, car elle nous enrichit davantage en tant que personnes et professionnels de l'éducation. II est très important de promouvoir toutes ces actions, non seulement pour nous, futurs enseignants / éducateurs, mais aussi pour la société en elle-même et pour promouvoir la culture des citoyens. (Narrative, 2D)

\section{Considérations finales}

Cette expérience de formation centrée sur la mise en œuvre d'initiatives d'activisme a muni les futurs enseignants de capacités pour l'action et la transformation sociale et environnementale. Devant réaliser une intervention auprès d'un public-cible de leur choix, les participants à l'étude ont fini par construire une connaissance plus approfondie sur des thèmes qu'ils connaissaient peu, devenant ainsi des citoyens plus responsables dans la société, ressentant le besoin de changer de comportements et de mettre en œuvre des actions visant à sensibiliser d'autres citoyens de sorte que 
ceux-ci changent à leur tour, leurs comportements et commencent à agir de manière responsable dans la société où ils vivent. Ils ont reconnu la valeur ajoutée de ce type de dynamiques dans l'inculcation des valeurs de préservation de l'environnement et dans la promotion de connaissances sur divers thèmes de manière plus motivante et plus efficace. Les enseignants en formation initiale ont considéré que la promotion les valeurs de l'environnement chez les enfants est fondamentale, afin de leur permettre des changements de comportements et de valoriser les actions individuelles dans la société. Ainsi, il est compréhensible que tous les enseignants en formation aient l'intention de promouvoir des initiatives d'action sociopolitiques dans leur future pratique professionnelle. La planification de l'action sociopolitique a conduit les futurs enseignants à développer des compétences de base pour planifier une action et d'agir dans la société afin de promouvoir en elle des transformations sociales et environnementales. Lorsqu'ils ont été confrontés au besoin de concevoir et mettre en œuvre une action sociopolitique, les futurs enseignants ont dû faire face à de nombreux défis. Au-delà du choix du thème et des destinataires de l'action, les étudiants-professeurs ont été encouragés à penser et à choisir une forme d'intervention. C'est alors que leur manque de connaissances envers ce type d'activités, a fait émerger des difficultés au moment de planifier la forme d'intervention dans la mesure où ils n'avaient pas pris conscience de toutes les exigences nécessaires à sa concrétisation. Les plus grandes difficultés rencontrées par les participants étaient liées à la gestion de l'activité. En effet, certains répondants ont déclaré avoir eu des difficultés à déterminer les destinataires de leur action, à adapter leur intervention au public-cible ainsi qu'à obtenir un nombre raisonnable de participants dans leur action. Cependant, grand nombre de ces difficultés étaient issues de l'inexpérience au niveau de la planification de ce type d'intervention. D'une manière générale, selon les futurs enseignants, l'un des grands avantages associés aux projets d'action sociopolitique correspond à la possibilité de développer plusieurs capacités chez les participants. L'expérience de formation qui a fait l'objet de cette étude a permis à tous les groupes la mise en œuvre effective d'une action auprès d'un groupe-cible, se situant au-delà d'une simple simulation dans le contexte de la classe.

Comme le conclut Hodson (2014), nous croyons que les expériences d'action sociopolitique réalisées dans le cadre de la formation des enseignants peuvent fonctionner comme un levier pour permettre à ces futurs enseignants de participer au quotidien, d'une manière plus responsable et plus active et à encourager, à leur tour, le développement de ces compétences chez leurs futurs étudiants. Une formation de 
cette nature a donc un double impact, au niveau de la vie personnelle et professionnelle de chaque individu.

\section{Références Bibliographiques}

Bader, B., \& Laberge, Y. (2014). Activism in Science and Environmental Education: Renewing Conceptions About Science Among Students When Considering Socioscientific Issues. In L. Bencze \& S. Alsop (Eds), Activist Science and Technology Education (pp. 419-449). London: Springer.

Bardin, L. (2009). Análise de conteúdo. Lisboa: Edições 70.

Bencze, J.L., \& Sperling, E.R. (2012). Student-teachers as advocates for student-led research-informed socioscientific activism. Canadian Journal of Science, Mathematics \& Technology Education, 12(1), 62-85.

Blatt, E. N. (2014). Developing an "Activist Mentality" in an Environmental Science Course. In L. Bencze \& S. Alsop (Eds), Activist Science and Technology Education (pp. 451-464). London: Springer.

Caetano, A. P. (2004). A mudança dos professores pela investigação-ação. Revista Portuguesa de Educação, 17(1), 97-118.

Chawla, L. (2008). Participation and the Ecology of Environmental Awareness and Action. In A. Reid, B. B. Jensen, J. Nikel \& V. Somovska (Eds.), Participation and Learning - Perspectives on Education and the Environment, Health and Sustainability (pp.98-110). London: Springer.

Conde, M. (2014). Activism mobilising science. Ecological economics, 105, 67-77.

Fernandes, A, Gonçalves, F., Pereira, M. J., \& Azeiteiro, U.M. (2007). Educação ambiental: características, conteúdos, objetivos e atividades práticas. O caso Português. In F. Gonçalves, R. Pereira, U. Manuel de M. Azeiteiro e M. J. V. Pereira (Eds.), Atividades práticas em ciência e educação ambiental (pp. 1141). Lisboa: Instituto Piaget.

Galvão, C. (2005). Narrativas em Educação. Ciência e Educação, 11(2),327-345.

Hodson, D. (2014). Becoming Part of the Solution: Learning about Activism, Learning through Activism, Learning from Activism. In L. Bencze \& S. Alsop (Eds), Activist Science and Technology Education (pp. 67-98).. London: Springer. 
Hoeg, D., \& Bencze, L. (2014). Counter Cultural Hegemony: Student Teachers' Experiences Implementing STSE-Activism. In L. Bencze \& S. Alsop (Eds), Activist Science and Technology Education (pp.575-596).. London: Springer.

Karahan, E., \& Roehrig, G. (2015). Constructing Media Artifacts in a Social Constructivist Environment to Enhance Students' Environmental Awareness and Activism. Journal of Science Education and Technology, 24, 103-118.

Kemmis, S., \& McTaggart, R. (1982). The action research planner. Victoria: Deakin University Press.

Kerckhoff, A. S., \& Reis, G. (2014). Responsible Stewards of the Earth: Narratives of Youth Activism in High School (Science). In L. Bencze \& S. Alsop (Eds), Activist Science and Technology Education (pp.465-476). London: Springer.

Legardez, A., \& Simonneaux, L. (2004). Les conditions de la discussion dans l'enseignement des questions socialement vives. In M. Tozzi \& R. Etienne (Eds.), La discussion en éducation et en formation - un nouveau champ de recherches (pp. 95-119). Paris: L’Harmattan.

Lei $n . .11 / 87$, de 7 de Abril (Alterada pela Lei $n .0$ 13/2002, de 19 de Fevereiro - Lei de Bases do Ambiente).

Lester, B.T., Ma, L., Lee, O., \& Lambert, J. (2006). Social activism in elementary science education: a science, technology, and society approach to teach global warming. International Journal in Science Education, 28(4), 315-33.

Linhares, E., \& Reis, P. (2014). La promotion de l'activisme chez les futurs enseignants partant de la discussion de questions socialement vives. Revue Francophone du Développement Durable, 4, 80-93.

Pazos, M. S. (2002). Algunas reflexiones sobre la investigación-acción colaboradora en la educación. Revista Eletrónica de Enseñanza de las Ciencias, 1(1), 1-17.

Reis, P. (2008). As narrativas na formação de professores e na investigação em educação. NUANCES: estudos sobre Educação, 15(16), 17-34.

Reis, P. (2014a). Promoting students' collective socio-scientific activism: Teacher's perspectives. In L. Bencze \& S. Alsop (Eds.), Activism in science and technology education (pp. 547-574). London: Springer.

Reis, P. (2014b). Acción socio-política sobre custiones socio-científicas: reconstruyendo la formación docente y el currículo. Uni-Pluri/versidad, 14(2), 
http://aprendeenlinea.udea.edu.co/revistas/index.php/unip

Reis, P. \& Climent, N. (2012). Narrativas de profesores: reflexiones en torno al desarrollo personal y profesional. Sevilla: Universidade International de Andalucía

Roth, W.-M. (2009). On Activism and Teaching. Journal for Activist Science \& Technology Education, 1(2), 31-47.

Schusler, T. M. \& Krasny, M. E. (2015). Science and Democracy in Youth Environmental Action - Learning "Good" Thinking. In M. P. Mueller \& D. J. Tippins (Eds.), EcoJustice, Citizen Science and Youth Activism - Situated Tensions for Science Education (pp.363-384). London: Springer.

Simonneaux, J., \& Simonneaux, L. (2012). Educational configurations for teaching environmental socioscientific issues within the perspective of sustainability. Research in Science Education, 42(1), 75-94.

Simovska, V. (2008). Learning in and as Participation: A Case Study from HealthPromoting Schools. In A. Reid, B. B. Jensen, J. Nikel \& V. Somovska (Eds.), Participation and Learning - Perspectives on Education and the Environment, Health and Sustainability (pp.61-80). London: Springer. 


\section{Long Abstract (800 words)}

Informed collective action, also known as activism, oriented to solve society's problems, offers opportunities for life-long learning (in particular, about science and technology and their interactions with society and the environment) and citizens' education regarding responsible action and problem solving. To counteract an increasingly diminished participation of citizens resulting from the growing complexity of the issues affecting our society, it is important to invest in a teacher training oriented to the promotion of a socio-political action (activism) and a more fair and sustainable society (Hodson, 2014). Accordingly to Blatt (2014), the fact that students are involved (during their courses) in socio-political actions do not imply that they will develop an activist behaviour in their everyday lives; however, they are given opportunities to develop an "activist mentality" when they are encouraged to reflect on their behaviour and how their actions can contribute to the resolution of social problems.

Considering fundamental to develop more responsible and scientifically informed citizens, this action-research intended to: a) equip future teachers with the skills necessary for the design, implementation and evaluation of initiatives aimed at sociopolitical action on social acute questions; b) promote the participants' citizenship skills and their environmental awareness; c) identify the difficulties and potentialities of their involvement in such initiatives; and d) understand the impact of this type of educational experience in future teachers personal and professional development. The participants involved action-research were graduation students in Elementary Education (future teachers) attending a subject on Environment. The analysis of their narratives has shown the development of planning and social intervention skills through different types of initiatives. The implemented actions triggered the development of skills necessary for socio-political planning and action towards social and environmental transformation. The participants developed a deeper knowledge on social acute questions, becoming more aware and active citizens, feeling the need to change their own behaviour and to implement actions in order to mobilize other citizens for responsible collective actions in society. They recognized the added value of this type of initiative in the development of environmental values and knowledge in a more motivating and effective way. Participants established the connection between the promotion of environmental values and their future professional practice as teachers, considering important to educate children in order to change their individual behaviour and develop a sense of appreciation about the effect of their own actions. It was also possible to sensitize the actions' target audiences to act more responsibly in the environment. The socio-political initiatives were carried out in different contexts, as the practicum contexts (Primary Schools), Free Time Activity Centres, the Superior School of Education (the initial teacher training institution of the participants), and the Web 2.0. All teacher students considered that they intend to integrate socio-political action initiatives in their future professional practice.

When confronted with the task of designing and implementing a socio-political action the future teachers faced several challenges. The greatest difficulties were related with the planning of the initiative accordingly to the characteristics and interests of the target 
group. However, many of these difficulties resulted from his inexperience in planning this type of intervention. 